\title{
Pharmacotherapy Management for COVID-19 and Cardiac Safety: A Data Mining Approach for Pharmacovigilance Evidence from the FDA Adverse Event Reporting System (FAERS)
}

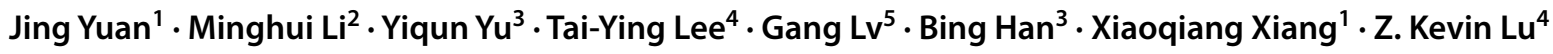

Accepted: 18 January 2021 / Published online: 10 February 2021

(c) The Author(s) 2021

\begin{abstract}
Background Several pharmacological agents, such as chloroquine/hydroxychloroquine, have been promoted for COVID19 treatment or pre-exposure prophylaxis. However, no comprehensive evaluation of the safety of these possible agents is available, and is urgently needed.

Objective The purpose of this study was to investigate the risks of cardiac adverse events associated with the possible pharmacotherapies for COVID-19, including certain antimalarial, antiviral, and antibiotic drugs.

Patients and Methods We conduced retrospective pharmacovigilance analyses of the US Food and Drug Administration Adverse Event Reporting System database. The reporting odds ratio (ROR), a data mining algorithm commonly used in pharmacovigilance assessment, was generated to quantify the detection signal of adverse events.

Results Among individuals without coronavirus infection from 2015 Q1 to 2020 Q1, increased risks for cardiac disorders were found for antiviral agents such as chloroquine/hydroxychloroquine (ROR: 1.68; 95\% confidence interval [CI] 1.66-1.70), lopinavir/ritonavir (ROR: 1.52; 95\% CI 1.39-1.66), and antibiotics such as azithromycin (ROR: 1.37; 95\% CI 1.30-1.44) and ceftriaxone (ROR: 1.92; 95\% CI 1.80-2.05). Increased serious cardiac adverse events, including myocardial infarction, arrhythmia, and cardiac arrest, were also reported for these drugs. Further analyses of individuals with coronavirus infections revealed that $40 \%$ of individuals receiving chloroquine/hydroxychloroquine reported serious cardiac adverse events. Two cases resulted in QT prolongations and one case resulted in cardiac arrest. Chloroquine/hydroxychloroquine and azithromycin contributed to all the QT prolongation and cardiac arrest cases.

Conclusions The current pharmacotherapies for COVID-19 are associated with increased risks of cardiac adverse events. Variations in the cardiac safety profiles of these pharmacotherapies were also observed. Clinicians should closely monitor patients with COVID-19, especially those at high risk, using chloroquine/hydroxychloroquine and azithromycin.
\end{abstract}

\section{Introduction}

Over five million cases of Coronavirus Disease 2019 (COVID-19), caused by the severe acute respiratory syndrome coronavirus 2 (SARS-CoV-2) [1], were reported worldwide as of 21 May, 2020 [2]. A large majority of individuals with COVID-19 have reported experiencing mild-tomoderate symptoms, including fever, cough, and fatigue [3],

Jing Yuan, Minghui Li, Yiqun Yu, Bing Han have contributed equally to the article.

Xiaoqiang Xiang

xiangxq@fudan.edu.cn

$\triangle$ Z. Kevin Lu

lu32@email.sc.edu

Extended author information available on the last page of the article

\section{Key Points}

Chloroquine/hydroxychloroquine may have contributed to a large proportion of cardiac disorders for COVID-19 treatment or pre-exposure prophylaxis.

The cardiac risks associated with the pharmacotherapies seem to be further elevated in patients with coronavirus infection, and close monitoring of cardiac risks is needed.

The potential pharmacotherapies are associated with serious cardiac adverse events, such as myocardial infarction, arrhythmia, and cardiac arrest. 
most of which do not require medical attention. However, COVID-19 can cause severe complications or even death in older adults or high-risk patients with concurrent chronic diseases [3]. Currently, there is no proven effective treatment against COVID-19. Infection control measures and supportive care, for example, supplemental oxygen and mechanical ventilatory support, are the main management strategies recommended by the current COVID-19 Treatment Guidelines [4]. With the rapid growth of the COVID-19 pandemic, there is an urgent need to identify existing medications deemed to be safe and effective with acceptable side effects, to attenuate infections and prevent severe complications in individuals with COVID-19.

Currently, three antiviral drugs-remdesivir, hydroxychloroquine, and chloroquine-have received emergency use authorization from the US Food and Drug Administration (FDA) in May 2020 [5]. These three drugs demonstrated antiviral properties against SARS-CoV-2 in an early in-vitro study [6]. Chloroquines showed benefits in patients hospitalized with COVID-19, based on two small clinical studies [7, 8]. Thereafter, on 23 March, 2020, the FDA allowed the emergency use of chloroquines in confirmed COVID-19 cases who were hospitalized. However, more recent research demonstrated no benefits of using chloroquines in treating COVID-19 infection [9, 10]. In addition to remdesivir, several antiviral agents such as lopinavir/ritonavir have been tested while showing uncertain benefits in treating COVID19 [4]. The combined use of antiviral agents and antibiotics, such as azithromycin and fluoroquinolones, has also been reported with mixed results.

While COVID-19 is the disease that primarily manifests in the respiratory system [11], cardiac disorders, such as coronary artery disease (CAD), heart failure (HF), and cerebrovascular disease, are common in hospitalized individuals with COVID-19 [3]. One potential mechanism is that myocardial cells have strong receptor angiotensin-converting enzyme II expression, which could be the target of SARSCoV-2 infection [12]. Other possible explanations include hypoxia and microthrombi, which cause vascular damage [13]. Considering the potential cardiac risks associated with some antiviral agents, however, pharmacotherapy may also play a role in the elevated risk of cardiac adverse events (AEs) observed in individuals with COVID-19. For example, chloroquine and hydroxychloroquine have long been proven to increase the risk of torsade de pointes (TdP) and cause QT prolongation [14], which further increases the patient's susceptibility to adverse cardiovascular outcomes and death during aggressive responses to COVID-19 infections. Risks of ventricular arrhythmia and cardiac arrests are also complicated by the incremental effects of azithromycin. After the emergence of severe cardiac AEs associated with hydroxychloroquine and chloroquine, the FDA cautioned against the use of investigational drugs outside the setting of a health system or clinical trial to avoid possible cardiovascular damage.

With the rapid growth of the COVID-19 pandemic and current promotion of certain pharmacological agents for COVID-19 treatments or pre-exposure prophylaxis, evidence on the cardiac risks of these possible therapies is urgently needed for clinicians to make informed decisions related to the benefits and risks of pharmacotherapies [15]. Therefore, the objective of this study was to investigate the association between the possible pharmacotherapies for COVID-19 and the risks of cardiac AEs.

\section{Methods}

\subsection{Study Design and Data Source}

We conducted a retrospective pharmacovigilance study using the FDA Adverse Event Reporting System (FAERS) database. The AEs reported for the potential pharmacotherapies for COVID-19 were extracted and queried from the first quarter of 2015 through the first quarter of 2020 . We included the study period from 2015 and forward because the goal of the study was to investigate the risks of cardiac AEs associated with the possible pharmacotherapies for COVID-19. Therefore, although the medications may not be used for COVID-19 before the end of 2019, the potential risks may still be possible for other indications and are worthy of evaluation. In addition, the FAERS data were limited since the COVID-19 pandemic, therefore, we included data from 2015 and forwards to evaluate the safety of the therapies. These drugs were commonly used in clinical settings or under investigation to treat patients with COVID-19 [16].

The FAERS is a post-marketing surveillance system that collects spontaneous AEs reported directly from consumers, healthcare professionals, pharmaceutical companies, or others [17]. The database contains nearly 20 million reports from 1969 to March 2020 [17]. The database contains detailed information on patient demographics, indications, medication use, outcomes, and sources of reports. All AEs were coded using Preferred Terms (PTs) in the Medical Dictionary for Regulatory Activities terminology [18]. The Medical Dictionary for Regulatory Activities hierarchy consists of five levels, from specific to broad categories. These coded terms are arranged in a hierarchy of five categories. The "System Organ Classes", grouped by disease etiology, manifestation site, or purpose, are the high-level group terms. The PTs, describing the disease symptoms or diagnosis, an indication, laboratory results, and procedures, are the low-level group terms and were used in the FAERS database [18]. 


\subsection{Identification of Exposures}

We searched the FAERS database to identify reports involving drug generic names, including chloroquine, hydroxychloroquine, lopinavir, ritonavir, ribavirin, umifenovir, tocilizumab, sarilumab, darunavir, favipiravir, azithromycin, moxifloxacin, ceftriaxone, piperacetazine, tazobactam, ampicillin, sulbactam, and tobramycin. A clinical pharmacist also reviewed the medications identified by the search queries. Oseltamivir was not included in the analysis because it was an investigational drug and not permitted for use outside of clinical trials. Drugs coded as suspect or concomitant use were also examined. For AEs reported in the first quarter of 2020, we further limited to those reports with coronavirus infection, to identify individuals with COVID-19.

\subsection{Identification of Cardiac Disorder}

To search for cardiac-related terms, we used the following PTs, including myocardial infarction, tachycardia, bradycardia, palpitations, cardiac failure, cardiac arrest, coronary artery disease, atrial fibrillation, and arrhythmia [19]. To understand the risks of overall cardiac AEs, we also searched the PTs listed under the System Organ Class of cardiac disorders in the Medical Dictionary for Regulatory Activities [18].

\subsection{Identification of Coronavirus Infection}

To identify patients with COVID-19, we searched reports involving "coronavirus infection" or "coronavirus test positive", in the first quarter of 2020. To test this data mining strategy, we also compared the number of individuals with coronavirus infection from 2015 Q1 through 2019 Q4. The number of individuals was fewer than 40 . In the first quarter of 2020, there were 311 individuals with coronavirus infection. Given the substantial increase in the number of infections, most of them should have COVID-19.

\subsection{Data Analyses}

To evaluate the risk of drug-associated AEs, we calculated the reporting odds ratio (ROR) [20], the data mining algorithm commonly used in a pharmacovigilance assessment. The RORs were calculated in the contingency $2 \times 2$ table, as described elsewhere [20]. We calculated the ROR for each drug of interest. If the lower bound of the $95 \%$ confidence interval (CI) of a ROR is greater than the threshold of 1.0 , then the signal of an $\mathrm{AE}$ is considered as significant, or the detected AE is associated with a statistically significantly higher number of reports following the use of the drug of interest, compared with those reports following the use of all other drugs [20].

\section{Results}

\subsection{Individuals Without Coronavirus Infection}

In the FAERS database from 2015 Q1 to 2020 Q1, a total of 6.12 million reports were identified. The number of individuals reporting the use of each drug was 347,034 for chloroquine/hydroxychloroquine, 7506 for lopinavir/ritonavir, 38,810 for ribavirin, 5615 for darunavir, 38,049 for tocilizumab/sarilumab, 10 for umifenovir, 11 for favipiravir, 26,093 for azithromycin, 8838 for moxifloxacin, 12,595 for ceftriaxone, 1478 for piperacetazine/tazobactam, 351 for ampicillin/sulbactam, and 11,456 for tobramycin (Fig. 1a).

Of 6.12 million reports, 288,232 reports of cardiac AEs were indented. Table 1 compared basic characteristics of FAERS reports. The total number of cardiac AEs were 24,618 (Fig. 1a, 7.09\%) for chloroquine/hydroxychloroquine, 501 (6.67\%) for lopinavir/ritonavir, 1311 (3.38\%) for ribavirin, $279(4.97 \%)$ for darunavir, $1683(4.42 \%)$ for tocilizumab/sarilumab, 2 (20\%) for umifenovir, 2 (18.18\%) for favipiravir, 1582 (6.06\%) for azithromycin, 839 (9.49\%) for moxifloxacin, 1045 (8.30\%) for ceftriaxone, $131(8.86 \%)$ for piperacetazine/tazobactam, 21 (5.98\%) for ampicillin/ sulbactam, and 239 (2.09\%) for tobramycin, respectively.

For chloroquine/hydroxychloroquine, the most frequently reported cardiac AaEs were palpitations (Fig. 2a, $n=3079$ ), cardiac failure $(n=3129)$, myocardial infarction [MI] $(n=$ $2964)$, tachycardia $(n=2859)$, and atrial fibrillation $[\mathrm{AF}](n$ = 2441). The RORs (95\% CI) associated with chloroquine/ hydroxychloroquine were statistically significant, including 1.68 (1.66-1.70) for overall cardiac disorders, 1.35 (1.28-1.42) for cardiac arrest, 1.45 (1.40-1.50) for MI, 1.73 (1.66-1.80) for tachycardia, 1.77 (1.67-1.87) for arrhythmia, $1.79(1.70-1.88)$ for bradycardia, $1.80(1.73-1.86)$ for cardiac failure, and 2.28 (2.05-2.53) for CAD. When chloroquine/hydroxychloroquine concomitantly with azithromycin, the RORs were still significant (Fig. 2b). This finding was also consistent with previous findings that azithromycin may be associated with increased cardiac risks [25, 26, 28]. Taken together, close monitoring for QTc prolongation is necessary for patients who are female, older, have a history of HF, or use chloroquine/hydroxychloroquine with azithromycin.

As shown in Fig. 2c, the most frequently reported cardiac AEs for lopinavir/ritonavir were cardiac failure $(n=82)$, MI $(n=77)$, palpitations $(n=56)$, tachycardia $(n=44)$, and $\mathrm{AF}(n=44)$. The significant ROR signals were 1.52 (1.39-1.66) for overall cardiac disorders, 1.70 (1.36-2.13) for MI, 2.23 (1.63-3.06) for arrhythmia, 2.19 (1.63-2.95) for bradycardia, 2.09 (1.68-2.60) for cardiac failure, and 1.57 (1.17-2.11) for AF. The signal for palpitations was detected for ribavirin (Fig. 2 d, ROR $=1.18,95 \% \mathrm{CI}$ 
Fig. 1 Frequency of cardiac disorders in the FDA Adverse Event Reporting System 2015 Q1 to 2020 Q1. a Demonstrates the proportion of cardiac disorders among individuals without coronavirus indications. b Demonstrates the proportion of cardiac disorders among individuals with coronavirus indications. AEs adverse events, $C Q$ chloroquine, $H C Q$ hydroxychloroquine, Sulbacta sulbactam, Tazo tazobactam

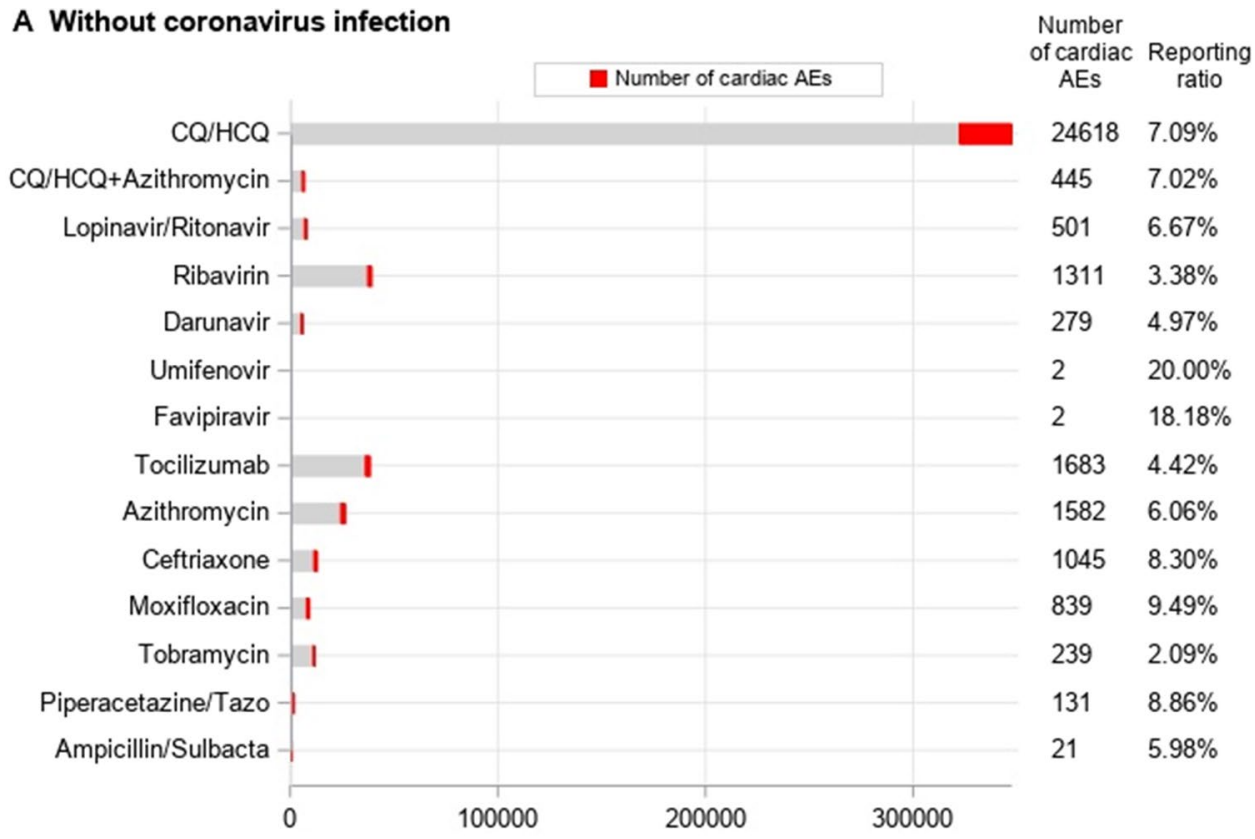

B With coronavirus infection

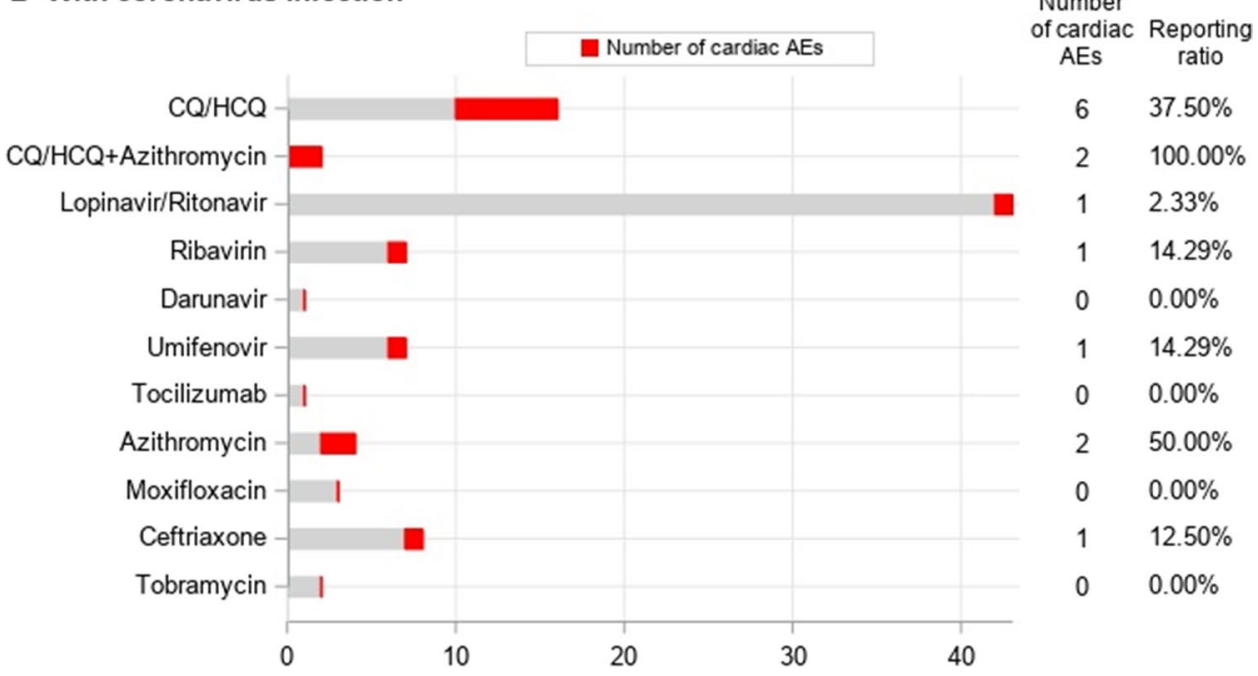

1.04-1.35). For darunavir (Fig. 2e), the ROR signals for $\mathrm{MI}$ and CAD were 2.95 (2.42-3.60) and 4.72 (2.79-7.98), respectively. For tocilizumab/sarilumab (Fig. 2f), the ROR signal for MI was 1.45 (1.30-1.62). For umifenovir and favipiravir, the number of events was too small to calculate RORs. For azithromycin only, the most frequently reported cardiac AEs were tachycardia (Fig. $2 \mathrm{~g}, n=212$ ), palpitations $(n=197)$, AF $(n=172)$, and cardiac failure $(n=$ 163). The RORs $(95 \%$ CI) was 1.37 (1.30-1.44) for overall cardiac disorders, 1.51 (1.26-1.80) for cardiac arrest, 1.64 (1.44-1.88) for tachycardia, 1.45 (1.26-1.67) for palpitations, 1.26 (1.02-1.55) for arrhythmia, 1.51 (1.23-1.86) for bradycardia, 1.19 (1.02-1.39) for cardiac failure, and 1.77 (1.52-2.06) for CAD, respectively. For ceftriaxone (Fig. 2h), the significant ROR signals were 1.92 (1.80-2.05) for overall cardiac disorders, 4.50 (3.88-5.23) for cardiac arrests, 3.43 (2.99-3.93) for tachycardia, 1.39 (1.03-1.90) for arrhythmia, 2.65 (2.15-3.27) for bradycardia, 1.67 (1.38-2.02) for cardiac failure, and 1.77 (1.42-2.19) for AF, respectively. For moxifloxacin (Fig. 2i), the RORs (95\% CI) were 2.23 (2.08-2.39) for overall cardiac disorders, 1.88 (1.43-2.47) for cardiac arrest, 2.32 (1.91-2.82) for tachycardia, 3.32 (2.61-4.22) for arrhythmia, 1.52 (1.10-2.11) for bradycardia, 1.86 (1.51-2.30) for cardiac failure, 1.51 (1.15-2.00) for AF, and 2.56 (1.45-4.52) for CAD. For piperacetazine/ tazobactam (Fig. 2j), the significant ROR signals were 2.06 (1.72-2.47) for overall cardiac disorders, 3.70 (2.29-5.96) for cardiac arrest, 5.15 (3.71-7.13) for tachycardia, 1.77 
Table 1 Patient characteristics in the FDA Adverse Event Reporting System (FAERS) database 2015 Q1 to 2020 Q1

\begin{tabular}{|c|c|c|c|c|c|c|}
\hline \multirow[t]{2}{*}{ Characteristics } & \multicolumn{3}{|c|}{ Without coronavirus infection } & \multicolumn{3}{|c|}{ With coronavirus infection ${ }^{\mathrm{a}}$} \\
\hline & $\begin{array}{l}\text { No cardiac disorders } \\
n(\%)\end{array}$ & $\begin{array}{l}\text { Cardiac disorders } \\
n(\%)\end{array}$ & $P$ value & $\begin{array}{l}\text { No cardiac disorders } \\
n(\%)\end{array}$ & $\begin{array}{l}\text { Cardiac disorders } \\
n(\%)\end{array}$ & $P$ value \\
\hline \multicolumn{3}{|l|}{ Age, years } & $<0.0001$ & & & $<0.0001$ \\
\hline $0-17$ & $7371(2.56)$ & $17,990(2.94)$ & & $11(3.86)$ & $10(38.46)$ & \\
\hline $18-44$ & $35,932(12.47)$ & $691,341(11.31)$ & & $38(13.33)$ & $3(11.54)$ & \\
\hline $45-64$ & $64,994(22.55)$ & $1,170,182(19.14)$ & & $56(19.65)$ & $3(11.54)$ & \\
\hline $65-75$ & $42,702(14.82)$ & $649,677(10.62)$ & & $31(10.88)$ & $2(7.69)$ & \\
\hline 75 and over & $40,274(13.97)$ & $540,490(8.84)$ & & $13(4.56)$ & $4(15.38)$ & \\
\hline Unknown & $96,959(33.64)$ & $2,883,488(47.15)$ & & $136(47.72)$ & $4(15.38)$ & \\
\hline \multicolumn{3}{|l|}{ Sex } & $<0.0001$ & & & 0.120 \\
\hline Female & $142,923(49.59)$ & $3,308,570(54.1)$ & & $116(40.70)$ & $16(61.54)$ & \\
\hline Male & $116,680(40.48)$ & $2,026,205(33.13)$ & & $133(46.67)$ & $8(30.77)$ & \\
\hline Unknown & $28,629(9.93)$ & $780,393(12.76)$ & & $36(12.63)$ & $2(7.69)$ & \\
\hline \multicolumn{3}{|l|}{ Year } & $<0.0001$ & & & \\
\hline 2015 & $49,631(17.22)$ & $1,038,146(16.98)$ & & & & \\
\hline 2016 & $48,839(16.94)$ & $1,055,895(17.27)$ & & & & \\
\hline 2017 & $51,771(17.96)$ & $1,090,998(17.84)$ & & & & \\
\hline 2018 & $60,304(20.92)$ & $1,290,049(21.1)$ & & & & \\
\hline 2019 & $61,198(21.23)$ & $1,285,828(21.03)$ & & & & \\
\hline 2020 & $16,489(5.72)$ & $354,252(5.79)$ & & $285(100)$ & $26(100)$ & \\
\hline \multicolumn{3}{|l|}{ Country } & $<0.0001$ & & & 0.0357 \\
\hline USA & $145,599(50.51)$ & $4,461,211(72.95)$ & & $136(47.72)$ & $18(69.23)$ & \\
\hline Non-USA & $142,633(49.49)$ & $1,653,957(27.05)$ & & $149(52.28)$ & $8(30.77)$ & \\
\hline \multicolumn{3}{|c|}{ Reporter's type of occupation } & $<0.0001$ & & & 0.0584 \\
\hline Consumer & $112,593(39.06)$ & $3,034,172(49.62)$ & & $113(39.65)$ & $4(15.38)$ & \\
\hline Lawyer & $5266(1.83)$ & $81,556(1.33)$ & & $0(0.00)$ & $0(0.00)$ & \\
\hline Physician & $71,291(24.73)$ & $1,260,021(20.6)$ & & $94(32.98)$ & $10(38.46)$ & \\
\hline $\begin{array}{l}\text { Other health profes- } \\
\text { sional }\end{array}$ & $71,276(24.73)$ & $1,121,967(18.35)$ & & $50(17.54)$ & $7(26.92)$ & \\
\hline Pharmacist & $19,830(6.88)$ & $453,270(7.41)$ & & $15(5.26)$ & $4(15.38)$ & \\
\hline Unknown & $7976(2.77)$ & $164,182(2.68)$ & & $0(0.00)$ & $1(3.85)$ & \\
\hline
\end{tabular}

${ }^{\text {a}}$ Reports identified with "Coronavirus infection" in the FAERS from 1 January to 31 March, 2020

(0.84-3.72) for bradycardia, $3.26(2.20-4.84)$ for AF, and 4.39 (2.93-6.58) for CAD. The signal for cardiac failure was detected for ampicillin/sulbactam (Fig. 2k, ROR $=2.74$, 95\% CI 1.13-6.62).

\subsection{Individuals with Coronavirus Infection}

During the first quarter of 2020, a total of 311 individuals with coronavirus infection were identified. The number of patients reporting the use of the following drugs were 16 for chloroquine/hydroxychloroquine (Fig. 1b), 43 for lopinavir/ritonavir, 7 for ribavirin, 1 for darunavir, 1 for tocilizumab/sarilumab, 7 for umifenovir, 4 for azithromycin, 3 for moxifloxacin, 8 for ceftriaxone, and 2 for tobramycin, respectively. Among them, six drugs were reported with cardiac AEs, including chloroquine/hydroxychloroquine $(n=$
5, 31.25\%), lopinavir/ritonavir $(n=1,2.33 \%)$, ribavirin ( $n$ $=1,14.29 \%)$, umifenovir $(n=1,14.29 \%)$, azithromycin $(n$ $=2,50.00 \%)$, and ceftriaxone $(n=1,12.50 \%)$.

Of 311 individuals with coronavirus infections, 26 patients reported cardiac AEs. As shown in Table 1, ten or $38.5 \%$ of individuals were dominantly aged under 17 years $(p<0.001)$. For chloroquine/hydroxychloroquine, as shown in Fig. 3a, the most frequently reported cardiac AEs included cardiac arrest $(n=1)$, tachycardia $(n=2)$, and QT prolongation $(n=2)$. When chloroquine/hydroxychloroquine was combined with azithromycin, cardiac arrest and QT prolongation were reported (Fig. 3b). Two cases of QT prolongation were reported for individuals using chloroquine/ hydroxychloroquine.

Cardiac arrest $(n=1)$ and tachycardia $(n=1)$ were reported for azithromycin with strong signals (Fig. 3f). For 
Fig. 2 Reporting odds ratio (ROR) of cardiac adverse events among individuals without coronavirus infection. The ROR and the $95 \%$ confidence interval (CI) of each cardiac adverse event following the use of a chloroquine/hydroxychloroquine (CQ/HCQ), b CQ/HCQ plus azithromycin, c lopinavir/ritonavir, $\mathbf{d}$ ribavirin, e darunavir, $\mathbf{f}$ tocilizumab/sarilumab, $\mathbf{g}$ azithromycin, $\mathbf{h}$ ceftriaxone, $\mathbf{i}$ moxifloxacin, $\mathbf{j}$ piperacetazine/tazobactam, and $\mathbf{k}$ ampicillin $\backslash$ sulbactam are provided in the adjacent tables. Note that the vertical red bar shows the line of ROR $=1$. RORs $(95 \% \mathrm{CI})$ in bold represent the significant signals of cardiac adverse events. Data source: FDA Adverse Event Reporting System 2015 Q1-2020 Q1

ceftriaxone, the two cases of cardiac AEs were tachycardia and cardiac arrest (Fig. 3g). The signals of cardiac AEs were not strong for lopinavir/ritonavir $(n=1)$, ribavirin $(n=1)$, and umifenovir $(n=1)$.

\section{Discussion}

This study found that the existing potential pharmacotherapies are associated with increased risks of overall cardiac disorders. We also found that nearly half of the individuals with coronavirus infections reported cardiac disorders following pharmacotherapies. In particular, chloroquine/ hydroxychloroquine was reported to develop serious cardiac AEs, including tachycardia, cardiac arrest, and QT prolonged in the overall sample. In addition, individuals with coronavirus infections had further elevated risks of cardiac disorders compared with the overall sample. We also observed variations in the cardiac safety profiles of the pharmacotherapies available for patients with COVID-19. Therefore, in clinical practice, clinicians should be aware of the elevated cardiac AEs in patients with COVID-19 and weigh up both the benefits and harms of the therapies. Continued pharmacovigilance is needed on cardiac disorders of other possible pharmacotherapies for COVID-19 to improve patient health outcomes.

The increased cardiac risks reported in patients with COVID-19 using chloroquine/hydroxychloroquine and azithromycin can be explained by drug-induced QTc prolongation, a measure of delayed ventricular repolarization [21]. It can be fatal enough to result in sudden death. Excessive QTc prolongation with a corrected interval $>500 \mathrm{~ms}$ can predispose the myocardium to the development of early arrhythmia, which in turn can trigger re-entrant tachycardia as the deadly Tdp. As a surrogate marker for developing Tdp, QTc prolongation has been an established side effect of class I and III anti-arrhythmic medications. This analysis revealed that azithromycin and hydroxychloroquine were associated with QT prolongations and cardiac arrest, consistent with a recent multi-national cohort study, in which chloroquine/

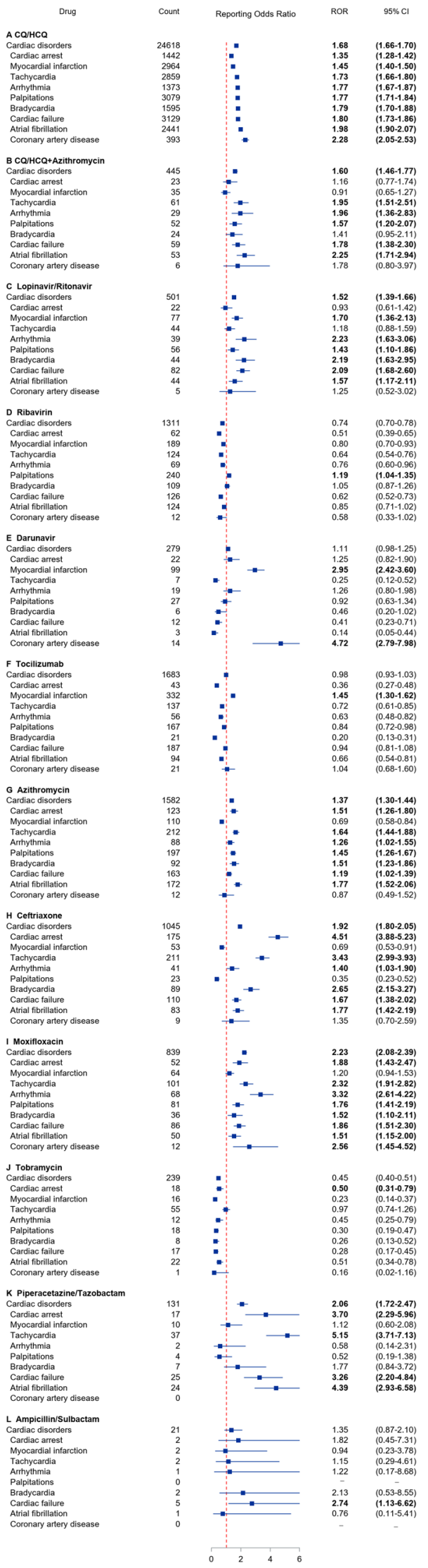




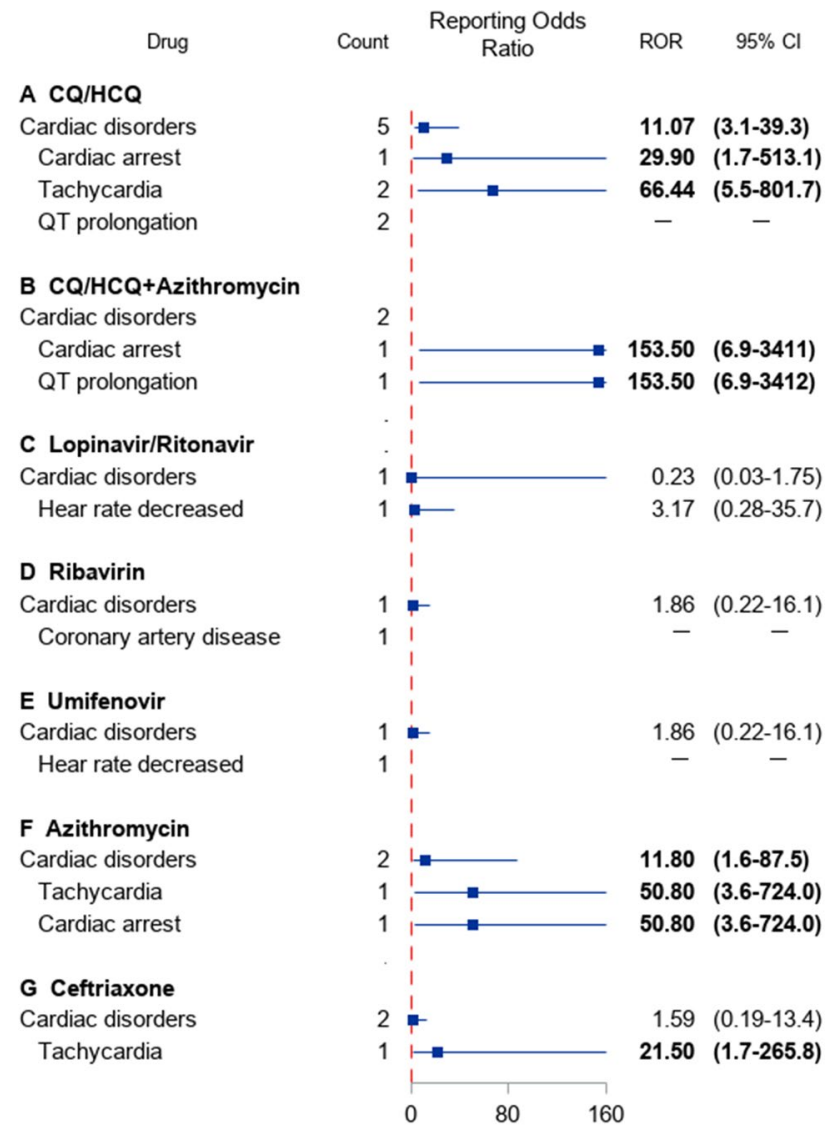

Fig. 3 Reporting odds ratio (ROR) of cardiac adverse events among individuals with coronavirus infection. Individuals with Preferred Terms of "Coronavirus infection". The ROR and the 95\% confidence interval (CI) of each cardiac adverse event following the use of a chloroquine/hydroxychloroquine (CQ/HCQ), b CQ/HCQ plus azithromycin, c lopinavir/ritonavir, $\mathbf{d}$ ribavirin, e umifenovir, $\mathbf{f}$ azithromycin, and $\mathbf{g}$ ceftriaxone are provided in the adjacent tables. Note that the vertical red bar shows the line of ROR $=1$. RORs $(95 \%$ CI) in bold represent the significant signals of cardiac adverse events. Data source: FDA Adverse Event Reporting System 2020 Q1

hydroxychloroquine with or without azithromycin increased the risks of arrhythmias and in-hospital mortality.

Hydroxychloroquine specifically was consistent with increased risks of arrhythmia-related events such as palpitations, tachycardia, and a further complication of AF. For antiretrovirals used for COVID-19, most cardiac AEs are statistically significant in the group of lopinavir/ritonavir, especially for high-risk individuals with pre-existing cardiac injury or comorbidities such as HF and prior MI, resulting in its early stop in $13.8 \%$ of patients because of unfavorable reactions. In comparison with lopinavir/ritonavir, other antiretrovirals are not as prominent while it is important to weigh the risk-benefit ratio when selecting the best therapy.
For instance, darunavir should be avoided in individuals with a history of CAD or prior MI as it significantly increases the risks of cardiac deterioration. Selected antibacterial agents for the treatment of COVID-19 have not shown promising outcomes but significantly increased risks of developing unwanted cardiac AEs. These findings strongly suggest caution in the use of certain medications with respect to cardiac adverse risks, particularly those patients at a high risk of cardiac disorders, either for COVID-19 treatment or pre-exposure prophylaxis.

Frequently used azithromycin and moxifloxacin have consistently shown increased risks of cardiovascular damage and mortality, especially among elderly individuals with pneumonia [23]. While beta-lactams such as ceftriaxone and zosyn cause more harms than benefits in patients who are more likely to experience tachycardia, and have symptoms of $\mathrm{AF}, \mathrm{HF}$, and cardiac arrest [24]. The risk of developing TdP is not solely based upon one medication but a cumulative effect of multiple risk factors such as female sex, increasing age, genetic disposition, and the use of multiple medications that can prolong QTc intervals, affect the metabolism of another QTc-prolonging drug, or cause renal/hepatic dysfunction [25, 26]. Hence, it is important to evaluate the patient demographic and clinical profile before administering possible pharmacotherapies for COVID-19 to minimize cardiac disorders.

In addition to drug-induced cardiac AEs, SARS-CoV-2 itself may cause heart inflammation, and is notorious in worsening pre-existing cardiovascular conditions in patients with myocarditis, HF, and arrhythmia [11]. Different etiologies of acute cardiac injury resulting from COVID-19 may include right-sided HF secondary to hypoxia-mediated pulmonary hypertension, worsening of coronary perfusion due to reduced oxygen-blood saturation, and most severely, a hyperacute systematic inflammatory response syndrome like sepsis, especially seen in older patients with multiple underlying chronic diseases admitted to the intensive care units. Moreover, a problem concerning potassium levels has arisen in patients with COVID-19 as a result of the interaction of SARS-CoV2 with the renal artery stenosis system [27]. Hypokalemia is known to increase the susceptibility of patients to arrhythmia and heart rhythm irregularities. Here, we found that chloroquine/hydroxychloroquine was associated with higher risks of cardiac arrhythmias. Therefore, it is recommended for the management of arrhythmia to optimize electrolyte concentrations through appropriate supplementation and fluids, avoiding high-risk medications, and closely monitoring the electrocardiograms of patients taking QTc-prolonging agents. 
This study has some strengths worth mentioning. The FAERS database includes a large amount of cardiac AEs of possible pharmacotherapies for COVID-19 reported worldwide. This enables researchers to examine cardiac AEs and compare to those in patients with COVID-19. This study further provides early signals for health professionals regarding adverse cardiovascular reactions during the selection of specific medications to be utilized or placed in future guidelines while ensuring maximal efficacy and patient safety. Onsite clinical judgment and incorporation of individualized patient factors are also important for optimizing treatment outcomes for patients with COVID-19.

This study possesses some limitations that should be considered. First, most case reports submitted were based upon personal observations and did not have a commonly agreed pathological pattern and treatment preference, leading to inconsistent data quality and potentially incomplete reports with missing information. Second, the reporting to the FAERS database is voluntary and can be easily biased. Depending on the variable criteria used for the selection of events, some cases may not be interpreted as incidence and can be omitted. Third, the sample size of patients with COVID-19 was relatively limited owing to the unavailability of the latest data in the FAERS database. Fourth, we could not exclude the possibility of unmeasured confounding factors, such as disease severity and coexisting conditions and complications, which may over- or under-estimate the association with potential pharmacotherapies and the risk of cardiac AEs. Hence, further studies are needed to make a causal link between drug exposure and cardiac AEs. Last, we cannot exclude the possibility that some patients with coronavirus infection actually had other conditions, such as influenza, rather than COVID-19. By comparing the number of individuals in the previous quarters, more than $90 \%$ of individuals identified in the 2020 Q1 are expected to have COVID-19.

\section{Conclusions}

The current pharmacotherapies for COVID-19, particularly chloroquine/hydroxychloroquine and azithromycin, are associated with increased risks of cardiac AEs. Variations in the cardiac safety profiles of these pharmacotherapies were also observed. The choice of pharmacotherapy should be based on demographic and clinical profiles. Cardiac monitoring should be employed for patients with COVID-19, especially those at high risk.

\section{Appendix}

See Tables 2 and 3.

Table 2 List of preferred terms

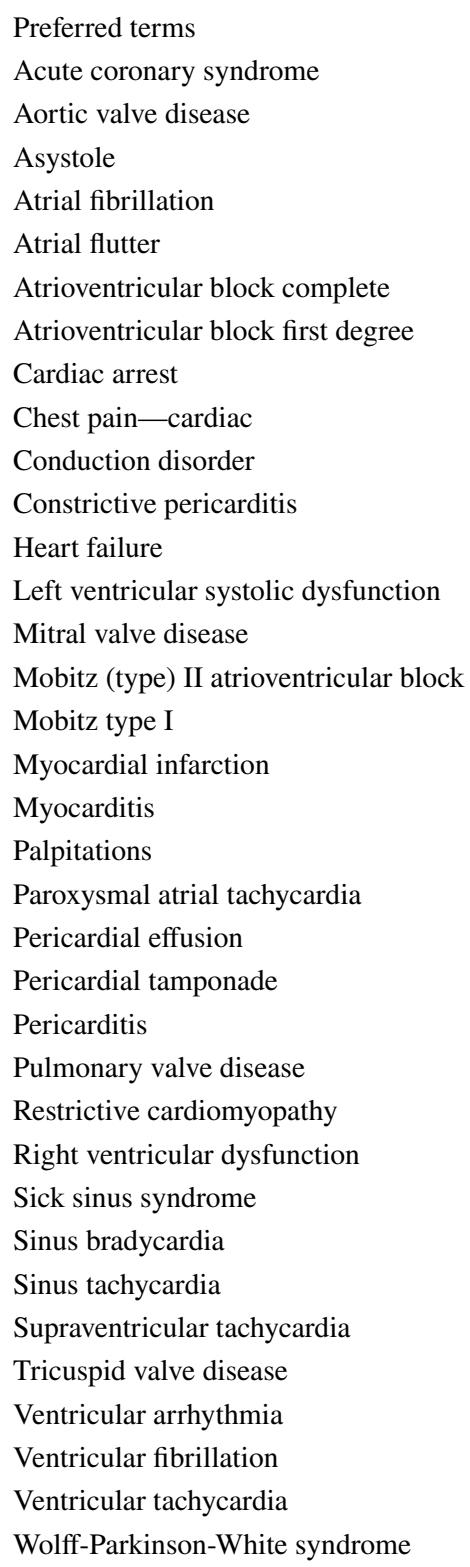

Preferred terms

Acute coronary syndrome

Aortic valve disease

Asystole

Atrial fibrillation

Atrial flutter

Atrioventricular block complete

Atrioventricular block first degree

Cardiac arrest

Chest pain-cardiac

Conduction disorder

Constrictive pericarditis

Heart failure

Left ventricular systolic dysfunction

Mitral valve disease

Mobitz (type) II atrioventricular block

Mobitz type I

Myocardial infarction

Myocarditis

Palpitations

Paroxysmal atrial tachycardia

Pericardial effusion

Pericardial tamponade

Pericarditis

Pulmonary valve disease

Restrictive cardiomyopathy

Right ventricular dysfunction

Sick sinus syndrome

Sinus bradycardia

Supraventricular tachycardia

Tricuspid valve disease

icular tachycardia 
Table 3 Number of individuals with coronavirus infection in the FDA Adverse Event Reporting System database, 2015 Q1-2020 Q1

\begin{tabular}{|c|c|}
\hline Quarter & $\begin{array}{l}\text { Number of individu- } \\
\text { als with coronavirus } \\
\text { infection }\end{array}$ \\
\hline 2020Q4 & 311 \\
\hline 2019Q4 & 32 \\
\hline 2019Q3 & 36 \\
\hline 2019Q2 & 37 \\
\hline 2019Q1 & 43 \\
\hline 2018Q4 & 38 \\
\hline 2018Q3 & 40 \\
\hline 2018Q2 & 40 \\
\hline 2018Q1 & 39 \\
\hline 2017Q4 & 12 \\
\hline 2017Q3 & 9 \\
\hline 2017Q2 & 21 \\
\hline 2017Q1 & 23 \\
\hline 2016Q4 & 14 \\
\hline 2016Q3 & 10 \\
\hline 2016Q2 & 9 \\
\hline 2016Q1 & 7 \\
\hline 2015Q4 & 2 \\
\hline 2015Q3 & 0 \\
\hline 2015Q2 & 6 \\
\hline 2015Q1 & 6 \\
\hline
\end{tabular}

\section{Declarations}

Funding This research did not receive any funding.

Conflicts of Interest/Competing interest Jing Yuan, Minghui Li, Xiaoqiang Xiang, Tai-Ying Lee, Gang Lv, Yiqun Yu, Bing Han, and Z. Kevin Lu have no conflicts of interest that are directly relevant to the content of this article.

Ethics approval This research does not involve human subjects. It is not required to obtain institutional review board approval.

Consent to participate Not applicable.

Consent for Publication Not applicable.

Availability of data and material Data are available upon reasonable request.

Code availability SAS codes are available upon reasonable request.

Authors' contributions Concept and design: JY, ML, XX, ZKL. Acquisition, analysis, or interpretation of data: JY, ZKL, GL, XX, ML. Drafting of the manuscript: JY, ZKL, GL, ML. Critical revision of the manuscript for important intellectual content: JY, ZKL, GL, ML, YY, BH, XX. Statistical analysis: JY, ML. Administrative, technical, or material support: ZKL, XX, BH. Supervision: ZKL, XX, BH.
Open Access This article is licensed under a Creative Commons Attribution-NonCommercial 4.0 International License, which permits any non-commercial use, sharing, adaptation, distribution and reproduction in any medium or format, as long as you give appropriate credit to the original author(s) and the source, provide a link to the Creative Commons licence, and indicate if changes were made. The images or other third party material in this article are included in the article's Creative Commons licence, unless indicated otherwise in a credit line to the material. If material is not included in the article's Creative Commons licence and your intended use is not permitted by statutory regulation or exceeds the permitted use, you will need to obtain permission directly from the copyright holder. To view a copy of this licence, visit $\mathrm{http} / / /$ creativecommons.org/licenses/by-nc/4.0/.

\section{References}

1. World Health Organization. Naming the coronavirus disease (COVID-19) and the virus that causes it. Available from: https:// wwwwhoint/emergencies/diseases/novel-coronavirus-2019/ technical-guidance/naming-the-coronavirus-disease-(covid2019)-and-the-virus-that-causes-it. Accessed 20 Jan 2021.

2. Dong E, Du H, Gardner L. An interactive web-based dashboard to track COVID-19 in real time. Lancet Infect Dis. 2020;20(5):533-4.

3. Novel CPERE. The epidemiological characteristics of an outbreak of 2019 novel coronavirus diseases (COVID-19) in China. Zhonghua Liu Xing Bing Xue Za Zhi. 2020;41(2):145.

4. Health NIo. Coronavirus disease 2019 (COVID-19) treatment guidelines 2020. https://www.covid19treatmentguidelines.nih. gov/whats-new/. Accessed 15 Apr 2020.

5. US Food and Drug Administration. Fact sheet for health care providers emergency use authorization (EUA) of hydroxychloroquine sulfate supplied from the strategic national stockpile for treatment of Covid-19 in certain hospitalized patients. 2020. https:// www.fda.gov/emergency-preparedness-and-response/mcm-legal -regulatory-and-policy-framework/emergency-use-authorization. Accessed 15Apr 2020.

6. Wang M, Cao R, Zhang L, Yang X, Liu J, Xu M, et al. Remdesivir and chloroquine effectively inhibit the recently emerged novel coronavirus (2019-nCoV) in vitro. Cell Res. 2020;30(3):269-71.

7. Gao J, Tian Z, Yang X. Breakthrough: chloroquine phosphate has shown apparent efficacy in treatment of COVID-19 associated pneumonia in clinical studies. Biosci Trends. 2020;14(1):72-3.

8. Gautret P, Lagier J-C, Parola P, Meddeb L, Mailhe M, Doudier $\mathrm{B}$, et al. Hydroxychloroquine and azithromycin as a treatment of COVID-19: results of an open-label non-randomized clinical trial. Int J Antimicrob Agents. 2020;56(1):105949.

9. Tang W, Cao Z, Han M, Wang Z, Chen J, Sun W, et al. Hydroxychloroquine in patients with mainly mild to moderate coronavirus disease 2019: open label, randomised controlled trial. BMJ. 2020;369:m1849.

10. Rosenberg ES, Dufort EM, Udo T, Wilberschied LA, Kumar J, Tesoriero J, et al. Association of treatment with hydroxychloroquine or azithromycin with in-hospital mortality in patients with COVID-19 in New York State. JAMA. 2020;323(24):2493-502.

11. Zheng Y-Y, Ma Y-T, Zhang J-Y, Xie X. COVID-19 and the cardiovascular system. Nat Rev Cardiol. 2020;17(5):259-60.

12. Zou X, Chen K, Zou J, Han P, Hao J, Han Z. Single-cell RNAseq data analysis on the receptor ACE2 expression reveals the potential risk of different human organs vulnerable to $2019-\mathrm{nCoV}$ infection. Front Med. 2020;14(2):185-92.

13. Li Y, Wang M, Zhou Y, Chang J, Xian Y, Mao L, et al. Acute cerebrovascular disease following COVID-19: a single center, retrospective, observational study. 2020. 
14. Burrell ZL Jr, Martinez AC. Chloroquine and hydroxychloroquine in the treatment of cardiac arrhythmias. N Engl J Med. 1958;258(16):798-800.

15. Giudicessi JR, Noseworthy PA, Friedman PA, Ackerman MJ, editors. Urgent guidance for navigating and circumventing the QTc-prolonging and torsadogenic potential of possible pharmacotherapies for coronavirus disease 19 (COVID-19). In: Mayo Clinic Proceedings. Elsevier; 2020. https://doi.org/10.1016/j. mayocp.2020.03.024

16. Sanders JM, Monogue ML, Jodlowski TZ, Cutrell JB. Pharmacologic treatments for coronavirus disease 2019 (COVID-19): a review. JAMA. 2020;323(18):1824-36.

17. U.S. Food and Drug Administration. Questions and Answers on FDA's Adverse Event Reporting System (FAERS). Accessed at:https://www.fda.gov/drugs/surveillance/questions-and-answe rs-fdas-adverse-event-reporting-system-faers. Accessed 15 Apr 2020.

18. MedDRA. Introductory Guide MedDRA Version 21.1. Accessed at: https://www.meddra.org/how-to-use/support-documentation/ english. Accessed15 Apr 2020.

19. Cherepanov V, Fortmann SD, Hyun Kim M, Marciniak TA, Litvinov $\mathrm{O}$, Mihalev $\mathrm{K}$, et al. Annual adverse event profiles after clopidogrel, prasugrel, and ticagrelor in the food and drug administration adverse event reporting system. Eur Hear J Cardiovasc Pharmacother. 2018;4(2):69-71.

20. van Puijenbroek EP, Bate A, Leufkens HG, Lindquist M, Orre R, Egberts AC. A comparison of measures of disproportionality for signal detection in spontaneous reporting systems for adverse drug reactions. Pharmacoepidemiol Drug Saf. 2002;11(1):3-10.

21. Saleh M, Gabriels J, Chang D, Kim BS, Mansoor A, Mahmood E, et al. Effect of chloroquine, hydroxychloroquine and azithromycin on the corrected QT interval in patients with SARS-CoV-2 infection. Circ Arrhythm Electrophysiol. 2020;13(6):e008662.

22. Mehra MRDS, Ruschitzka F, Patel AN. Hydroxychloroquine or chloroquine with or without a macrolide for treatment of COVID19: a multinational registry analysis. Lancet. 2020. https://doi. org/10.1016/S0140-6736(20)31180-6.

23. Mortensen EM, Halm EA, Pugh MJ, Copeland LA, Metersky M, Fine MJ, et al. Association of azithromycin with mortality and cardiovascular events among older patients hospitalized with pneumonia. JAMA. 2014;311(21):2199-208.

24. Lohiya G-S, Tan-Figueroa L, Krishna V. Piperacillin-induced immune hemolysis presenting with tachycardia and cardiac arrest. Case Rep Med. 2011;2011:816497.

25. Lehmann MH, Hardy S, Archibald D, Quart B, MacNeil DJ. Sex difference in risk of torsade de pointes with d, 1-sotalol. Circulation. 1996;94(10):2535-41.

26. Trinkley KE, Lee Page R, Lien H, Yamanouye K, Tisdale JE. QT interval prolongation and the risk of torsades de pointes: essentials for clinicians. Curr Med Res Opin. 2013;29(12):1719-26.

27. Chen D, Li X, Song Q, Hu C, Su F, Dai J, et al. Assessment of Hypokalemia and Clinical Characteristics in Patients With Coronavirus Disease 2019in Wenzhou, China. JAMA Netw Open. 2020;3(6):e2011122. https://doi.org/10.1001/jamanetworkopen .2020 .11122 .

28. Lu ZK, Yuan J, Li M, Sutton SS, Rao GA, Jacob S, et al. Cardiac risks associated with antibiotics: azithromycin and levofloxacin. Expert Opin Drug Saf. 2015;14(2):295-303. https:// doi.org/10.1517/14740338.2015.989210 ((Epub 2014 Dec 10. PMID: 25494485; PMCID: PMC4404501)).

\title{
Authors and Affiliations
}

\author{
Jing Yuan ${ }^{1} \cdot$ Minghui $\mathrm{Li}^{2} \cdot$ Yiqun $\mathrm{Yu}^{3} \cdot$ Tai-Ying Lee $^{4} \cdot \mathrm{Gang} \mathrm{Lv}^{5} \cdot \mathrm{Bing} \mathrm{Han}^{3} \cdot \mathrm{Xiaoqiang} \mathrm{Xiang}^{1} \cdot$ Z. Kevin $\mathrm{Lu}^{4}$ \\ 1 Department of Clinical Pharmacy, School of Pharmacy, \\ Fudan University, 826 Zhangheng Road, Pudong, \\ Shanghai 201203, People's Republic of China \\ 2 University of Tennessee Health Science Center, Memphis, \\ TN, USA \\ 4 University of South Carolina, 715 Sumter Street, CLS \\ Building 311, Columbia, SC 29208, USA \\ 5 General Surgery Department, 1st Medical Center of PLA \\ General Hospital, Beijing, China
}

3 Department of Pharmacy, Minhang Hospital, Fudan University, Shanghai, People's Republic of China 